\title{
Microbial keratitis in Sudanese contact lens users
}

\section{Letter to editor}

Contact lenses have become a popular replacement for eyeglasses in both developed and developing countries. In certain circumstances, however, undesirable responses to contact lenses can occur, and a range of microorganisms, such as bacteria, fungi, and free-living amoebae, can cause all sorts of eye infections. Contact lenses are an optical device that can be used to cure severe refractive defects as well as some medical conditions. Contact lenses may only be used for many years without creating problems if they are made of high-quality materials, are properly shaped, and are well fitted. The ocular effects range from slight annoyance to potentially blinding corneal ulceration and blindness. ${ }^{1}$ Microbial keratitis (MK) is a category of ophthalmic infectious diseases caused by bacteria, fungus, and protozoa that injure the cornea. It has the potential to cause ocular morbidity and dysfunction. ${ }^{2}$ Microbial keratitis is an infrequent but devastating side effect of using contact lenses that impacts approximately 5 individuals out of 10,000. Because of the large number of CL users worldwide, rare diseases with significant morbidity have major public health consequences. ${ }^{3}$ As a result, keratitis is considered an eye emergency that requires immediate and efficient antibiotic treatment to prevent irreparable vision loss. ${ }^{4}$

A total of 150 samples of contact lens aqueous solutions of $\mathrm{Al}$ Yarmouk University College students were used in the investigation. Bacteria were identified from eighty-four specimens' aqueous solutions, whereas sixty-six samples showed no growth. Pseudomonas aeurogenosa was the bacterium most often detected, contributing for $39 \%$ of all isolates, followed by Staphlycoccus aureus (32\%), Staphlycoccus epidermidis (12\%), and E.coli (6\%). Before wearing glasses, users who washed their hands with detergent soap had one isolated type of bacteria $83(55.3 \%)$, whereas users who did not wash their hands had a various types of bacterial growth $67(44.7 \%)$.The participants in the study were divided into two age groups (18-22) and (18-22). (23-26) with a $\mathrm{p}$ value of 0.05 , the study discovered a connection between age and the most identified microorganisms with a $p$ value of ( 0.001$)$. The plurality of eye lenses $(145 \%)$ were utilized for cosmetic reasons, while only $5(3.3 \%)$ were used for medical reasons. With a $\mathrm{p}$ value of 0.0052 , the research revealed a relationship between lens use and the majority of bacteria isolated. With a $p$ value of 0.001 , the study found a relationship between lens quality and the majority of bacteria. The preponderance of eye lenses (141, or $94 \%)$ was genuine, while the rest were counterfeit $(9$, or $6 \%)$. In terms of contact lens kind and quality, our research found that those who wear original contact lenses are more susceptible to bacterial infection. Microbial growth was seen in $56 \%$ of research participants, with a $\mathrm{P}$ value of $<0.05$. The most preventive component was found to be high compliance with lens care procedures, whereas inadequate compliance with hand washing before wearing lenses was associated to a high contamination incidence among study participants. The

\author{
Volume 9 Issue 3 - 202 I
}

\author{
Abduwahab Abakar Fadil,' Mohammed \\ Ahmed Ibrahim Ahmed, ${ }^{2}$ Magdi Babikir \\ Omer,' Nahla Ahmed Mohammed \\ Abdelrahman,' Nassreldeen Khalid \\ Abdelrahman ${ }^{3}$ \\ 'Al Yarmouk University College, Sudan \\ ${ }^{2}$ Nile Valley University, Sudan \\ ${ }^{3}$ University of AI Fashir, Sudan
}

Correspondence: Mohamed Ahmed Ibrahim Ahmed, Department of microbiology, Nile Valley University, Sudan, Emailmohammedabukleewa@gmail.com

Received: August 28, 202I | Published: September 09, 2021

plurality of eye lenses (145\%) were used for aesthetic purposes among our research respondents with a significant bacterial contamination rate, with 79 out of 150 indicating bacterial growth with a $P$ value of 0.052 . The most frequent age group linked with bacterial keratitis in the current study was under 25 years old, with a $\mathrm{P}$ value of 0.05 for bacterial growth, and P.aeruginosa was the most common bacterial isolate identified, followed by S.epidermidis.

\section{Acknowledgments}

None.

\section{Conflicts of interest}

The authors declare that there is no conflict of interest.

\section{References}

1. Review. Major Review Complications of Contact Lens. 2017.

2. Ezisi, Chinyelu N, Chimdia E Ogbonnaya, et al. Microbial Keratitis-A Review of Epidemiology, Pathogenesis, Ocular Manifestations, and Management. Nigerian Journal of Ophthalmology. 2018;26(1):13.

3. Stapleton, Fiona, Lisa Keay, et al. The Incidence of Contact Lens-Related Microbial Keratitis in Australia. Ophthalmology. 2008;115(10):16551662

4. Bartimote, Christopher, John Foster, et al. The Spectrum of Microbial Keratitis: An Updated Review. The Open Ophthalmology Journal. 2020(13). 